\title{
Social participation of patients with multiple sclerosis
}

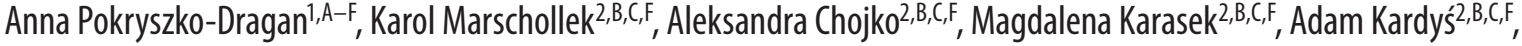 \\ Paweł Marschollek ${ }^{2, B, C, F}$, Ewa Gruszka ${ }^{1, B, C, F}$, Marta Nowakowska-Kotas ${ }^{1, B-D, F}$, Sławomir Budrewicz ${ }^{1, A, E, F}$ \\ ${ }^{1}$ Clinical Department of Neurology, Wroclaw Medical University, Poland \\ 2 Students' Scientific Club, Department of Neurology, Wroclaw Medical University, Poland \\ A - research concept and design; $\mathrm{B}$ - collection and/or assembly of data; $\mathrm{C}$ - data analysis and interpretation; \\ $\mathrm{D}$ - writing the article; $\mathrm{E}$ - critical revision of the article; $\mathrm{F}$ - final approval of the article
}

\section{Address for correspondence}

Anna Pokryszko-Dragan

E-mail:annapd@interia.pl

\section{Funding sources}

None declared

\section{Conflict of interest}

None declared

Received on May 17, 2019

Reviewed on June 26, 2019

Accepted on December 12, 2019

Published online on April 28, 2020

Cite as

Pokryszko-Dragan A, Marschollek K, Chojko A, et al.

Social participation of patients with multiple sclerosis.

Adv Clin Exp Med. 2020;29(4):469-473.

doi:10.17219/acem/115237

DOI

10.17219/acem/115237

Copyright

Copyright by Author(s)

This is an article distributed under the terms of the

Creative Commons Attribution 3.0 Unported (CC BY 3.0)

(https://creativecommons.org/licenses/by/3.0/)

\begin{abstract}
Background. The effect multiple sclerosis (MS) has on the social functioning and integration of patients has been recently considered as an important factor of the disease.

Objectives. To assess social participation of MS patients with regard to demographic and disease-related variables.

Material and methods. The study comprised 201 MS patients: 140 women, 61 men, aged 24-69 years. The World Health Organization Disability Assessment Schedule (WHODAS 2.0) was applied to assess the aspects of social functioning and the Beck Depression Inventory (BDI) was applied to evaluate the level of depression. Disease duration, degree of disability in Expanded Disability Status Scale (EDSS), most disabling symptoms and type of treatment were determined. WHODAS 2.0 scores (total and within particular domains) and their relationships with age, gender, disease-related variables and level of depression were analyzed.

Results. The results of WHODAS 2.0 for $27.4 \%$ of patients exceeded the $90^{\text {th }}$ percentile compared to the population norms (with the highest scores for "getting around" and "participation in society" domains). The results of BDI and WHODAS 2.0 were strongly correlated $(p<0.001 ; \beta=0.73)$ and mobility impairment was related to both of them ( $p<0.001 ; \beta=-0.12$ and 0.25 , respectively). Other disabling symptoms were associated with scores in domains "understanding and communicating", "getting around" and "participation in society".

Conclusions. Social participation of the MS patients is affected by the impact of disease and associated with depression. Particular symptoms of neurological deficit (motor and visual impairment, fatigue) influence social functioning more than general disease-related variables.
\end{abstract}

Key words: depression, disability, multiple sclerosis, social participation 


\section{Introduction}

Multiple sclerosis (MS) is a chronic, multiphasic disorder of the central nervous system. The complex background of the disease includes dysfunction of immune-mediated mechanisms, which is affected by interacting genetic predisposition and environmental factors. Multifocal damage to myelin and axonal loss within the brain and spinal cord may result in a wide range of symptoms and in accumulating physical and mental disability.

The most typical MS onset occurs in young adults and long-lasting course of the disease affects further stages of life. Therefore, MS impact upon the patients' social functioning constitutes an essential problem. ${ }^{1}$ Multiple sclerosis may influence the patients' ability to fulfill family and social roles according to the their expectations ${ }^{2}$ and may cause barriers to successful employment. ${ }^{3}$ The patients may prioritize self-care, health-promoting and daily activities to leisure and other aspects of social functioning. ${ }^{4}$ They may also feel stigmatized and isolated, which further affects their involvement in community activities. 5,6

Within recent years, the effect MS has on social participation and integration of the patients has been recognized as an important multidimensional factor of the disease. ${ }^{7}$ According to the World Health Organization (WHO) and International Classification of Functioning, Disability and Health (ICF), participation can be defined as engagement in major life activities in the lived environment and involvement in life situations. ${ }^{8,9}$ The evaluated dimensions of participation may include the frequency and range of activities, their importance and associated self-efficacy or satisfaction. ${ }^{5,10}$ The concept of social participation in MS patients has been already elaborated in some studies, ${ }^{1,2,4,10,11}$ which differed in analyzed issues and their measures.

The aim of the study was to assess the assessment of social functioning and participation in MS patients with regard to the demographic and disease-related variables.

\section{Material and methods}

Consecutive patients with MS diagnosed according to McDonald's criteria, ${ }^{12}$ consulted in the outpatient clinic at the Department of Neurology during January-March 2018, who had regular follow-ups documented in the medical records, were included in the study. Exclusion criteria comprised relapse of the disease or switch to another type of treatment within the preceding 3 months, as well as cognitive impairment which would interfere with filling out the self-administered questionnaires.

The studied group consisted of 201 patients: 140 women and 61 men aged 24-69 years (mean: 41.3 years). A relapsing-remitting (RRMS) course of disease was diagnosed in $91.5 \%$ and a secondary progressive (SPMS) course in $8.5 \%$. All the patients with RRMS were being treated with disease-modifying therapies (DMTs): 115 - interferon beta, 22 - glatiramer acetate, 24 - dimethylfumarate, 18 - fingolimod, 3 - natalizumab.

The assessment of social functioning was carried out using the World Health Organization Disability Assessment Schedule (WHODAS 2.0). ${ }^{13}$ Beck Depression Inventory $(\mathrm{BDI})^{14}$ was used to evaluate the level of depression. The self-administered questionnaire was also applied, and included questions concerning the patients' major complaints (symptoms perceived as most disabling) and their perception of the effectiveness and tolerability of treatment. Data on MS duration and its course, disease-modifying treatment and degree of disability (measured in Expanded Disability Status Scale - EDSS $)^{15}$ were obtained from the medical records.

The project of the study was approved by Bioethical Committee of Wroclaw Medical University. All the subjects gave their informed consent to participate in the study.

The total result of WHODAS 2.0 was calculated and compared to population norms. Scores in particular domains were presented as percentages. Relationships were analyzed between WHODAS 2.0 results and age, gender, duration of MS, EDSS, most disabling symptoms, type of medication used and BDI.

Statistical analysis was performed using STATISTICA v. 13.0 (StatSoft Polska, Cracow, Poland), with a significance level $\alpha=0.05$. Categorical variables were presented as numerical and percentage values and continuous ones - as the mean and standard deviation (SD) or median and interquartile range. Spearman's rank correlation was used to assess relationship between WHODAS 2.0 total score and BDI score and general linear models were conducted to establish predictors of WHODAS 2.0 results (total score and scores in each domain).

\section{Results}

In the studied group, MS duration ranged from 1 to 39 years (mean: 10.07) and EDSS from 1.0 to 7.0 (median: 3, Q1-Q3: 2-4). Mobility problems, fatigue and visual dysfunction were most common symptoms perceived by the patients as most disabling (Table 1). A total of 158 subjects (78.6\%) considered their treatment as effective, 88 (43.8\%) noticed side effects of DMTs.

The results of WHODAS 2.0 for $27.36 \%$ of patients were above $90^{\text {th }}$ percentile comparing to the population norms. Mean scores in particular domains are presented in Fig. 1.

Beck Depression Inventory score ranged from 0 to 63 (median: 7, Q1-Q3: 2-16); 64 (31.84\%) patients scored more than 13 , which indicates depressive symptoms (Table 2).

Total score of WHODAS 2.0 or scores in particular domains showed no significant relationships with age and gender of the patients. No significant relationships were found between WHODAS 2.0 results and MS duration or EDSS. 


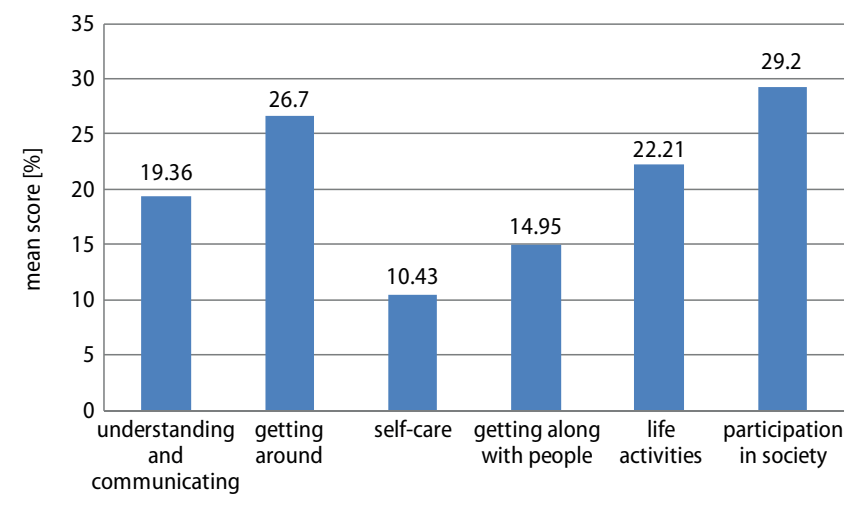

Fig. 1. Mean score in each WHODAS 2.0 domain (as a percentage of total amount of points possible to score)

WHODAS 2.0 - the World Health Organization Disability Assessment Schedule.

Table 1. Major complaints of MS patients (symptoms reported as most disabling). A patient could report more than 1 symptom

\begin{tabular}{|l|c|}
\hline \multicolumn{1}{|c|}{$\begin{array}{c}\text { Symptoms reported as most } \\
\text { disabling }\end{array}$} & $\begin{array}{c}\text { Number (percentage) } \\
\text { of patients }\end{array}$ \\
\hline Mobility problems & $80(39.8)$ \\
\hline Fatigue & $48(23.9)$ \\
Visual deficit & $35(17.4)$ \\
\hline Headache/vertigo & $28(13.9)$ \\
\hline Musculoskeletal pain/stiffness & $28(13.9)$ \\
\hline Hand/foot numbness & $22(10.9)$ \\
\hline Upper limb weakness & $13(6.5)$ \\
\hline Urinary incontinence & $10(5)$ \\
\hline Memory problems & $9(4.5)$ \\
\hline
\end{tabular}

MS - multiple sclerosis

Table 2. Distribution of BDI scores in the MS patients. Total score indicates: minimal depression (0-13), mild depression (14-19), moderate depression (20-28), severe depression (29-63)

\begin{tabular}{|l|c|}
\hline \multicolumn{1}{|c|}{ BDI score } & $\begin{array}{c}\text { Number (percentage) } \\
\text { of patients }(\mathrm{N}=201)\end{array}$ \\
\hline $0-13$ & $137(68.2)$ \\
\hline $14-19$ & $29(14.4)$ \\
\hline $20-28$ & $26(12.9)$ \\
\hline $29-63$ & $9(4.5)$ \\
\hline
\end{tabular}

BDI - Beck Depression Inventory.

Beck Depression Inventory and WHODAS scores were strongly correlated $(\mathrm{p}<0.01 ; \mathrm{R}=0.73)$ and the problem with mobility as the most disabling symptom was a predictor for both of them $(\mathrm{p}<0.001 ; \beta=-0.12$ and $\beta=0.26$, respectively). Specific symptoms claimed as the most disabling appeared to be the risk factors for higher score particularly in WHODAS domains. All significant associations are shown in Table 3.

No significant relationships were found between WHODAS 2.0 score and the type of medication used in MS therapy or the presence of side effects of treatment.
Table 3. Significant correlations between WHODAS 2.0 scores (total and in particular domains), most disabling symptoms and BDI score in the studied group of MS patients

\begin{tabular}{|c|}
\hline Total WHODAS 2.0 score \\
\hline - Mobility problems as most disabling symptom $(p<0.001 ; \beta=0.26)$ \\
\hline "Understanding and communicating" domain \\
\hline $\begin{array}{l}\text { - } \text { BDI score }(p<0.001 ; \beta=0.56) \\
\text { - Visual deficit as most disabling symptom }(p=0.006 ; \beta=0.16) \\
\text { - Fatigue as most disabling symptom }(p=0.016 ; \beta=0.14)\end{array}$ \\
\hline "Getting around" domain \\
\hline $\begin{array}{l}\text { - } \text { BDI score }(p<0.001 ; \beta=0.46) \\
\text { - Mobility problems as most disabling symptom }(p<0.001 ; \beta=0.43) \\
\text { - Musculoskeletal pain/stiffness as most disabling symptom }(p=0.04 ; \\
\beta=0.12)\end{array}$ \\
\hline "Participation in society" domain \\
\hline $\begin{array}{l}\text { - } \text { BDI score }(p<0.001 ; \beta=0.65) \\
\text { - Mobility problems as most disabling symptom }(p<0.001 ; \beta=0.26) \\
\text { - Headache/vertigo }(p=0.02 ; \beta=0.13)\end{array}$ \\
\hline BDI score \\
\hline $\begin{array}{l}\text { - Score in "getting along with people" domain }(p<0.001 ; \beta=0.31) \\
\text { - Score in "life activities" domain }(p=0.03 ; \beta=0.19) \\
\text { - Score in "participation in society" domain }(p<0.001 ; \beta=0.41) \\
\text { - Mobility problems as most disabling symptom }(p=0.05 ; \beta=-0.12)\end{array}$ \\
\hline
\end{tabular}

\section{Discussion}

There is a wide range of instruments to evaluate social functioning and participation which were used in the studies focused on MS patients. ${ }^{1,4,711}$ We chose WHODAS 2.0 because of its validation for $\mathrm{MS}^{16}$ and availability in the Polish language version. WHODAS 2.0 questionnaire addresses the difficulties and problems perceived by a respondent in particular domains. In view of that, the scale results provide better insight in the aspects of the patients' selfefficacy and satisfaction than a pure measure of frequency or range of social activities. ${ }^{5,10}$

The findings in our group of MS patients, compared to the population norms, revealed a high percentage of responses with a score above the $70^{\text {th }}$ percentile. The highest scores were reached in the domains "participating in society" and "life activities" and moderate ones in "getting along with people" and "understanding and communicating". Thus, important aspects of social functioning seemed significantly affected in the studied group. The previous studies in this field ${ }^{1,2,4,7,11,17}$ also showed relevant findings for various measures of social participation, including maintaining relationships, employment status and community integration.

No correlations were found in our MS group between WHODAS 2.0 scores and demographic variables. In other studies, ${ }^{1,18}$ age was not directly related to social functioning measures, but appeared as an interacting factor, linked with quality of life indices and employment status. Hughes et al. ${ }^{7}$ reported better scores for females in social domain of Community Integration Questionnaire, but no other genderspecific relationships were described in this field. 
In the studied group, over $90 \%$ of patients had a relapsingremitting course of MS and were being treated with DMTs, which were perceived as effective by the majority of the subjects. Mean level of disability in EDSS in the whole group was relatively mild. Considering these apparently favorable outcomes of the disease, high scores of WHODAS 2.0 certainly deserve attention. Furthermore, there were no significant correlations between WHODAS 2.0 results and MS duration and EDSS. Such relationships were not found for types of DMTs or their side effects, either. Other authors ${ }^{2,7,11,17}$ observed links between disability score and measures of community integration, social participation and employment status. However, their studies used various tools to evaluate social functioning, and they also differed in the sample size.

On the contrary to general indices of MS course, particular symptoms regarded by our patients as most disabling showed significant correlations with WHODAS 2.0 results. The most frequent major complaints (motor impairment, fatigue and visual dysfunction) correlated with scores in all the main domains of WHODAS, while less frequently reported headaches, vertigo, stiffness and pain were related to aspects of mobility and participating in social activities. Mobility impairment, fatigue and occasionally also visual impairment have been indeed shown to affect social participation, employment status and related satisfaction. ${ }^{3,19,20}$ Many authors ${ }^{2,4,7,17,18}$ highlighted the role of cognitive impairment which would have greater impact upon patients' social functioning than physical disability. Only a small percentage of our patients counted cognitive decline among their major complaints. Our methods did not include objective measures of cognitive performance, because its reliable assessment would require widening the questionnaire, possibly discouraging the patients from responding. However, we did consider the role of depression in the evaluation of social functioning issues. Indeed, almost $1 / 3$ of our patients had a BDI score indicating depressive symptoms and a significant correlation was found between the results of BDI and WHODAS. Although other studies ${ }^{2,7}$ reported less frequent depressive symptoms assessed with BDI or Hospital Anxiety and Depression Scale (HADS), their authors confirmed relevant influence of depression upon measures of social participation. ${ }^{7,10}$ It has been highlighted that apart from disease-related variables, social functioning of MS patients is affected by many interacting factors, including personal and environmental ones. ${ }^{1,3,10,20}$

Overall, our findings, which were obtained with specific and validated tools, show a relevant prospect of social participation in a representative group of MS patients, with contributing factors. Limitations of the study include homogeneity of the studied group (predominating patients with RRMS were probably more likely both to participate in social activities and to respond to the questionnaire than those with SPMS) and only single evaluation of the analyzed issues (without considering dynamics of the disease over time). These aspects could be addressed in the further studies planned in this field. Furthermore, other factors possibly affecting social participation of MS patients (including cognitive performance and level of education, socioeconomic status, etc.) should be also considered in the analyses.

\section{Conclusions}

Social functioning and participation of MS patients are substantially affected by the impact of the disease and show an association with depression. Particular symptoms of neurological deficit (motor and visual impairment, fatigue) seem to influence social functioning more than general disease-related variables. These aspects deserve attention during individualized follow-ups of MS patients.

\section{ORCID iDs}

Anna Pokryszko-Dragan (D) https://orcid.org/0000-0002-5203-112X Karol Marschollek (D) https://orcid.org/0000-0001-9093-180X Aleksandra Chojko (D) https://orcid.org/0000-0003-1233-887X Magdalena Karasek (D) https://orcid.org/0000-0002-1066-0876 Adam Kardyś (D) https://orcid.org/0000-0001-8230-1904

Paweł Marschollek (D) https://orcid.org/0000-0003-4588-309X

Ewa Gruszka (D) https://orcid.org/0000-0002-9800-5461

Marta Nowakowska-Kotas (D) https://orcid.org/0000-0002-3173-8337

Sławomir Budrewicz (D) https://orcid.org/0000-0002-2044-6347

\section{References}

1. Mikula P, Sc M, Nagyova I, et al. Social participation and health-related quality of life in people with multiple sclerosis. Disabil Health J. 2015;8(1):29-34. http://dx.doi.org/10.1016/j.dhjo.2014.07.002

2. Hakim AE, Bakheit AMO, Bryant TN, et al. The social impact of multiple sclerosis - a study of 305 patients and their relatives. Disabil Rehabil. 2000;22(6):288-293. http://www.tandfonline.com/doi/full/ 10.1080/096382800296755

3. Messmer Uccelli M, Specchia C, Miller D, Battaglia M. Factors that influence the employment status of people with multiple sclerosis: A multi-national study. J Neurol. 2009;256:1989-1996.

4. Plow MA, Finlayson M, Ont OTR, Gunzler D, Heinemann AW. Correlates of participation in meaningful activities among people with multiple sclerosis. J Rehabil Med. 2015;47:538-545.

5. Sparling A, Stutts LA, Sanner H, Eijkholt MM. In-person and online social participation and emotional health in individuals with multiple sclerosis. Qual Life Res. 2017;26(11):3089-3097.

6. Cook JE, Germano AL, Stadler G. An exploratory investigation of social stigma and concealment in patients with multiple sclerosis. Int J MS Care. 2016;18(2):78-84.

7. Hughes AJ, Hartoonian N, Parmenter B, et al. Cognitive impairment and community integration outcomes in individuals living with multiple sclerosis. Arch Phys Med Rehabil. 2015;96(11):1973-1979. http:// dx.doi.org/10.1016/j.apmr.2015.07.003

8. World Health Organization. International classification of functioning, disability and health. 2001. https://www.who.int/classifications/ icf/en/.

9. Resnik L, Plow MA. Measuring participation as defined by the International Classification of Functioning, Disability and Health: An evaluation of existing measures. Arch Phys Med Rehabil. 2009;90:856-866.

10. Yorkston KM, Kuehn CM, Johnson KL, Ehde DM, Jensen MP, Amtmann D. Measuring participation in people living with multiple sclerosis: A comparison of self-reported frequency, importance and self-efficacy. Disabil Rehabil. 2008;30(2):88-97.

11. Kwiatkowski A, Marissal J, Pouyfaucon M, Vermersch P, Hautecoeur P, Dervaux B. Social participation in patients with multiple sclerosis: Correlations between disability and economic burden. BMC Neurol. 2014; $14: 115$. 
12. Polman C, Reingold S, Banwell B, et al. Diagnostic criteria for multiple sclerosis: 2010 revisions to the McDonald criteria. Ann Neurol. 2011;69:292-302.

13. World Health Organization. http://www.who.int/classifications/icf/ WHODAS2.0_36itemsSELF.pdf. Accessed on December 15, 2017.

14. Beck AT, Ward CH, Medelson M, Mock J, Erbaugh J. An inventory for measuring depression. Arch Gen Psychiatry. 1961;4: 561-571.

15. Kurtzke J. Rating neurologic impairment in multiple sclerosis: an expanded disability status scale (EDSS). Neurology. 1983;33(11): 1444-1452.

16. Garin O, Ayuso-Mateos JL, Almansa J, et al. Validation of the "World Health Organization Disability Assessment Schedule, WHODAS-2" in patients with chronic diseases. Heal Qual Life Outcomes. 2010;8:51.

17. Cattaneo D, Lamers I, Bertoni R, Feys P, Jonsdottir J. Participation restriction in people with multiple sclerosis: Prevalence and correlations with cognitive, walking, balance, and upper limb impairments. Arch Phys Med Rehabil. 2017;98(7):1308-1315. http://dx.doi. org/10.1016/j.apmr.2017.02.015
18. Pompeii LA, Moon SD, McCrory DC. Measures of physical and cognitive function and work status among individuals with multiple sclerosis: A review of the literature. J Occup Rehabil. 2005;15(1):69-84.

19. Yorkston KM, Baylor C, Amtmann D. Journal of Communication Disorders Communicative participation restrictions in multiple sclerosis: Associated variables and correlation with social functioning. J Commun Disord. 2014;52:196-206. http://dx.doi.org/10.1016/j.jcomdis.2014.05.005

20. Hollingsworth $\mathrm{H}$, Gray DB. Structural equation modeling of the relationships between participation in leisure activities and community environments by people with mobility impairments. Arch Phys Med Rehabil. 2010;91(8):1174-1181. http://dx.doi.org/10.1016/j. apmr.2010.04.019 